\title{
Are Low Brain Derived Neurotrophic Factor Levels in the Blood a Biological Marker of Suicide Risk in Psychiatric Patients? A Systematic Review
}

\author{
Katerina Kavalidou ${ }^{\mathrm{a}}$, Diego De Leo ${ }^{\mathrm{a}, \mathrm{b}}$
}

\begin{abstract}
The functional polymorphism of the brain-derived neurotrophic factor (BDNF) in relation to suicidal behaviour has attracted a great deal of interest in recent research. Although genetic studies have indicated BDNF as a candidate gene in suicidal behaviour, no clear evidence exists for the role of BDNF levels in the blood of patients with mental illness who are at risk of suicide. Considering the ability of BDNF to cross the blood-brain barrier, the aim of the present study was to review evidence for a correlation between blood BDNF levels and suicidal behaviour among patients with psychiatric disorders. The systematic review that was performed (1966 - 2012) identified 64 studies as potential candidates for inclusion. After scrutiny, only seven studies appeared to focus on BDNF levels in plasma, serum and platelets. Studies consistently showed a significant decrease in BDNF levels among patients with previous suicide attempts, with the exception of one study, which included patients with schizophrenia. No significant differences were found between BDNF levels, gender and lethality of suicide attempts. Further evidence is required for which blood sample type to use when examining BDNF protein levels in terms of suicidal behaviour in mental illness sufferers, and more focus should be given to a potential blood BDNF threshold among patients at suicide risk.
\end{abstract}

Keywords: BDNF; Suicide risk; Mental disorders; Blood; Serum; Platelets

Manuscript accepted for publication December 28, 2012

${ }^{a}$ Australian Institute for Suicide Research and Prevention, National Centre of Excellence in Suicide Prevention, W.H.O. Collaborating Centre for Research and Training in Suicide Prevention, Griffith University QLD 4122, Australia

${ }^{\mathrm{b}}$ Corresponding author: Diego De Leo, Australian Institute for Suicide Research and Prevention, National Centre of Excellence in Suicide Prevention, W.H.O. Collaborating Centre for Research and Training in Suicide Prevention, Level 1, Building M24 Psychology, 176 Messines Ridge Road, Mt Gravatt campus, Griffith University QLD 4122, Australia. Email: d.deleo@griffith.edu.au

doi: http://dx.doi.org/10.4021/jnr171e

\section{Introduction}

Over the past decade many studies have focused on the pathophysiology of mental illness, in particular the role of the neurotrophin family, which has been implicated in stress-related mood disorders [1-6]. The brain-derived neurotrophic factor (BDNF), as a member of the neurotrophin family, is a protein with key roles in the peripheral nervous system (PNS) and central nervous system (CNS); it is involved in synaptic plasticity and functioning, and has been studied in relation to depression, schizophrenia, bipolar disorder and suicidal behaviour [7-14]. The deregulating role of BDNF has been extensively studied in stress-related mood disorders, since BDNF expression is proven to be stress-dependant $[1,15]$. A strong association between BDNF and major depression has been demonstrated, but much weaker effects have been shown with mild to moderate forms of self-reported depression [7, 16-19]. Although the relationship between BDNF and depression is not straightforward, the role of BDNF in the mechanism of antidepressant action has been a consistent finding $[1,15,20]$. BDNF plays a crucial role in the serotonergic system and is a target of a variety of antidepressants, including selective serotonin reuptake inhibitors (SSRIs), norepinephrine selective reuptake inhibitors (NESRIs), monoamine oxidase inhibitors (MAOIs), and atypical antidepressants $[1,20,21]$.

These studies have been used to support the "neurotropin hypothesis of depression", according to which impaired synaptic plasticity is the basis of the pathogenesis of the disorder; antidepressants improve synaptic plasticity and assist in altering neural networks to better model external stimuli and allow the individual to adapt to crisis situations [22-24]. An increased expression of BDNF has been reported among antidepressant-treated depressed patients compared to those who were antidepressant-naive, and BDNF levels were found to differ according to antidepressant treatment $[1,25]$.

Human genetic studies have shown reduced BDNF expression in individuals with suicidal behavior, regardless of psychiatric diagnosis, and a significant association of Met allele and risk of suicide attempts [1, 4, 22, 26-31]. Lee et al (2010) described a reduction of blood BDNF mRNA levels 
in patients with major depressive disorders (MDD) and recent suicide attempt, and implications of either the severity of depression or suicide behavior as BDNF regulators were sought. A moderator role for BDNF Val66Met polymorphism has been proposed in the case of childhood maltreatment leading to violent suicide attempts [4]. The BDNF rs6265 Val66Met marker is associated with reduced BDNF activity and Met allele carriers are at risk of suicidal behavior; yet there is no clear evidence of a direct association of Val66Met polymorphism with suicidal behavior or of an indirect effect through severity of depression $[27,29,32,33]$.

BDNF expression in suicide victims has been correlated with brain areas related to cognition and emotion, including the prefrontal cortex (PFC) and hippocampus [21, 34]. In terms of BDNF expression and its age regulation, adult and teenage suicides may have different pathogenic mechanisms, with teenage suicide subjects showing low BDNF expression in the PFC but not the hippocampus [35, 36].

While research on BDNF's role in gender-specific suicidal acts has not been extensive, studies have indicated the importance of BDNF Val66Met polymorphism in the development of major depressive disorder and BDNF expression in suicide victims, based on gender [10, 21, 37-39]. Kozicz et al (2008) have suggested a sex effect in BDNF regulation when examining the mid-brain area of suicide victims, and documented increased levels of BDNF in females compared to males. In terms of gender, Pregelj et al have indicated that the combined Met/Met and Met/Val genotypes of BDNF are risk factors for female completed suicide, especially in those women who have used violent suicide methods [40]. Furthermore, studies into cortisol and estrogen influences on gene expression have also documented BDNF differences among genders [41-43]. Gender differences in the BDNF regulation of suicide victims have been explored, but further clarification of gender effects is needed [37, 39, 40].

Although the significance of BDNF in blood is not yet clear [44], Pan et al. reported that intact BDNF in blood crosses the blood brain barrier (BBB) [45] and further animal studies have suggested that post-natally, platelet BDNF show changes similar to those found in cortical BDNF, highlighting a positive correlation between the two [45-47]. Furthermore Pillai et al have indicated the reflection of brain BDNF in blood by examining plasma BDNF and cerebrospinal fluid (CSF) correlation in a drug-naive sample with a first psychotic episode [48]. Based on the findings of parallel changes of brain and serum BDNF levels during depression, Elfving et al used a genetic rat model of depression and investigated BDNF in whole blood, serum, cerebrospinal fluid, hippocampus and frontal cortex [49]. While differences were found among the rat strains of this study in terms of the mature brain derived neurotrophic factor (mBDNF) regulation levels, BDNF was decreased at both mRNA and protein levels in Flinders Sensitive Line (FSL) rats; additionally negative correlations between free mBDNF levels in se- rum, whole blood and hippocampus were found [49]. Being one of the first studies reporting both brain and serum BDNF levels in a psychiatric disease model, the authors suggest further studies on the possible use of BDNF as a biomarker in depression [49].

Other mammalian species in addition to rats (mouse and pigs) were further investigated by Klein et al, in regard to the concentration of blood BDNF and its reflection to the brain tissue BDNF levels [50]. While the authors found a positive correlation of blood BDNF concentrations and the BDNF levels in hippocampus among rats and pigs, BDNF in mice was undetectable both in serum, plasma and whole blood [50]. In the context of animal studies and the role of BDNF, Klein et al demonstrated a close linkage between blood and brain BDNF and noted the potential use of blood BDNF measurements as predictor of BDNF levels in specific brain regions, more specifically hippocampus [50].

As such, blood BDNF levels might be a useful biological marker of suicide risk, considering the significant genetic findings of BDNF and suicidal behaviour [22, 31, 39].

\begin{abstract}
Aim
A variety of studies have considered evidence for BDNF deregulation in suicidal behaviour. However, the role of blood BDNF protein in the context of suicide has not been well established. Considering this, the aim of this study was to review articles on blood BDNF levels in patients, and clarify whether the findings of these studies are consistent with the previously reported correlation between genetic BDNF expression and suicidality.
\end{abstract}

\section{Materials and Methods}

A systematic literature search was undertaken of English language articles indexed in Pubmed, Web of Knowledge, Psych INFO and Scopus from 1966 till 2012, using the keywords "brain-derived", "neurotrophic", "factor", "suicide", and "attempt*". The reference sections of the publications found were scanned to identify additional studies that may have been missed. A total of 64 articles were collected and reviewed with regards to the selection criterion of: studies measuring and presenting findings about blood BDNF protein regulation and suicidal behaviour, among patients with different psychiatric disorders.

Genetic findings of BDNF regulation in suicide victims and attempters were excluded, as well studies not presenting blood BDNF protein levels related with suicidal behaviour as a measuring outcome. Based on the later, the study of KauerSant' Anna et al (2007) which measured serum BDNF levels among patients with bipolar disorder, related psychopathology and history of traumatic events (TE), was excluded from our review; the authors found differences among those 
with and without TE in terms of BDNF protein levels, but no presentation of the number of suicidal patients and the exact protein BDNF levels and suicide attempts correlation was given, rather than the level of significance (BDNF levels with history of suicide attempts, $\mathrm{P}=0.527$ ).

Further, articles written in languages other than English were excluded. A total of 7 articles were relevant to the study aim and met the selection and exclusion criteria. These are briefly summarised in supplementary data.

Considering the different blood measurements (serum, plasma, platelets) and methodology of the BDNF protein extraction, the lack of homogeneity regarding the wash-off period for medication, the length of inpatient hospitalisation and ethnic variability of study samples, a meta-analysis could not been conducted. Therefore the data synthesis in this review is descriptive.

\section{Results}

Based on the role of BDNF in schizophrenia, and the data on peripheral blood of patients $[25,51,52]$ the serum BDNF (sBDNF) levels of 126 schizophrenic patients and 96 healthy controls were examined in regards to the clinical phenotypes of: with/without suicide attempts; antipsychotic drug use; family tendency; disease onset before/after 25 years of age and different subtypes of schizophrenia [53]. No sBDNF differences were found between schizophrenic patients and control subjects $(\mathrm{F}=0.000, \mathrm{df}=1.220, \mathrm{P}=0.983)$, while catatonic schizophrenic patients were found to have lower SBDNF levels compared to paranoid and residual schizophrenic patients $(\mathrm{F}=3.272, \mathrm{df}=2.123, \mathrm{P}=0.041)$ [53]. No other differences were found between sBDNF and the remainder of the variables, and based on $8.7 \%$ of patients with previous suicide attempt, no significant sBDNF difference was noted for the clinical phenotype of suicide $(\mathrm{F}=0.040$, $\mathrm{df}=1.124, \mathrm{P}=0.841$ ) [53]. The absence of a significant association between BDNF and the clinical phenotypes measured was seen as being due to the potential limitations of: the short wash-out period of antipsychotics (1 week) and the unreliable knowledge of which medication (antipsychotics or antidepressants) was used prior the study period [53].

Considering the hypothesis that suicidality per se lowers the levels of BDNF, Deveci et al investigated 10 suicide attempters with adjustment disorders, 24 non-suicidal major depressive disorder (MDD) patients and 26 healthy individuals, for potential sBDNF differences [54]. Based on previous findings of decreased BDNF levels in MDD patients regardless of suicidal behavior, the authors highlighted the role of BDNF in suicidal behavior, since plasma BDNF levels were similarly low both in suicide attempters without major psychiatric disorder and the non-suicidal MDD group (supplementary data) [54]. According to the authors, the findings suggest BDNF as a biological marker of suicidal behavior among other stress-related mental disorders besides major depression [54]. The authors' interpretation of sBDNF decreased levels among suicide attempters was based on the possible down-regulation of BDNF expression from the decreased serotonin function and the severe stress associated with a previous suicide attempt [54].

In light of BDNF identification in platelets [55] and the difficulty in directly predicting peripheral BDNF levels to activity in the brain, the correlation of blood BDNF and suicide risk was examined through a direct internal jugular vein blood sampling [56]. Based on a sample of 19 unmedicated patients with MDD, BDNF was measured via the veno-arterial BDNF plasma concentration gradient [56]. The patients were assessed and grouped for depression severity with the Hamilton Depression Rating Scale (HAM-D), and for suicide risk with the Mini International Neuropsychiatric Interview (MINI). Results indicated: a significant reduced veno-arterial BDNF concentration gradient at medium to high suicide risk (supplementary data); a negative correlation between suicide risk and the internal jugular venous veno-arterial BDNF concentration gradient $((\mathrm{r}=0.721, \mathrm{P}=$ 0.001; veno-arterial $\mathrm{BDNF}=329-(27 \times$ suicide risk $))$; and, no association between the severity of depression and the internal jugular BDNF concentration gradient [5]. Based on the evidence of positive internal jugular veno-arterial BDNF concentration gradient (internal jugular BDNF plasma concentration $127 \pm 109 \%$ higher than the arterial concentration, $\mathrm{P}=0.04$ ), the authors noted an association between internal jugular venous BDNF overflow and suicide risk [56].

Carrying on the studies of biological markers in major depression and suicidal behaviour, some candidate markers for suicide were explored [57]. A variety of cytokines, neurotrophins and nitric oxide (NO) levels were examined about their possible correlation with the severity of depression and the lethality of suicide attempts among suicidal MDD patients and normal controls [57]. The study measured the lethality of previous suicidal acts (Weisman and Worden's RRR system and LSARS-II scale), Body Mass Index (BMI), and the severity of depression (using the 17-item Hamilton Rating Scale for Depression, HDRS). No significant differences in biological factors were found in regards to gender and the violent and non-violent suicide attempters, while BDNF in the suicidal depressed group was positively correlated with HDRS scores $(\gamma=0.370)$ [57].

According to the authors, the decreased levels of BDNF found in suicidal depressed patients implied a relationship with suicidal depression, since the other neurotrophins tested, such as $\beta$-NGF and IGF-1, did not differ significantly between the control group and the suicidal MDD group respectively ( $\beta$-NGF: $122.04 \pm 120.45 \mathrm{pg} / \mathrm{mL}$ vs. $109.48 \pm$ $113.21 \mathrm{pg} / \mathrm{mL}, \mathrm{F}=0.849, \mathrm{df}=96, \mathrm{P}=0.360 ;$ IGF-1: 358.22 \pm 525.17 vs. $427.37 \pm 255.48 \mathrm{pg} / \mathrm{mL}, \mathrm{F}=0.016, \mathrm{df}=96, \mathrm{P}$ $=0.901)$ [57].

In regards to the risk of suicide among depressed pa- 
tients, Kim et al examined plasma BDNF levels in patients with major depression disorder, with and without suicidal history, and healthy controls [58]. Further variables included were the severity of depression (using the HDRS), BMI, and lethality of suicide attempts which was measured using the RRR system and the LSARS-II [58]. Overall, no significant differences were found in: the mean HDRS score between suicidal and non-suicidal MDD patients $(\mathrm{P}=0.085)$; the correlation of BDNF levels with LSARS $(\gamma=0.118), \operatorname{RRR}(\gamma$ $=0.127)$ and HDRS scores $(\gamma=0.201)$ in suicidal MDD patients; the lethality of methods (highly lethal methods 361.72 $\pm 360.31 \mathrm{pg} / \mathrm{mL}$ vs less lethal methods $508.64 \pm 434.11 \mathrm{pg} /$ $\mathrm{mL}, \mathrm{t}=-1.046, \mathrm{P}=0.304)$; and BDNF levels between men and women $(742.71 \pm 500.27 \mathrm{pg} / \mathrm{mL}$ vs.719.81 $\pm 660.87 \mathrm{pg} /$ $\mathrm{mL}, \mathrm{t}=0.179, \mathrm{df}=92, \mathrm{P}=0.859)$ [58].

Mean BDNF level was significantly lower in the suicidal MDD group compared to the non-suicidal MDD and control groups; the proposed cut-off point of BDNF level between suicidal and non-suicidal depressed groups was $444.58 \mathrm{pg} / \mathrm{mL}$ [58]. Being one of the first studies presenting the lower plasma BDNF levels in suicidal MDD patients compared to normal controls and non-suicidal MDD patients, the study did not report any significant differences of BDNF levels among non-suicidal MDD and normal control group [58]. The authors additionally concluded that the unanticipated finding of no reduction of BDNF levels among the non-suicidal MDD group could potentially show BDNF as a biological marker of vulnerability to suicidal behaviour in depression, rather than as a marker of depression itself [58].

Lee et al (2007) examined plasma levels of BDNF in 77 MDD patients and 95 normal controls, and tested for correlations between $\mathrm{BDNF}$ levels, the clinical characteristics of depression subtypes and recent suicide attempt [59]. The study included the HDRS, BMI, and the Brief Psychiatric Rating Scale (BPRS) [59]. Mean plasma BDNF was significantly lower in MDD patients than normal controls (579.46 $\pm 414.25 \mathrm{pg} / \mathrm{mL}$ vs $819.20 \pm 347.05 \mathrm{pg} / \mathrm{mL}, \mathrm{t}=4.130, \mathrm{df}=$ $170, \mathrm{P}<0.01)$. No significant differences were found among groups for age, sex or BMI [59]. However based on BMI, differences were found between the normal controls and MDD patients without, and with, a suicide attempt, respectively $(22.0 \pm 3.0$ vs $21.7 \pm 2.6$ vs $20.3 \pm 2.7, \mathrm{~F}=3.963$, df $=171, \mathrm{P}=0.021$ ) [59]. Suicidal patients had significantly lower HDRS and BPRS scores than non-suicidal patients, and positive correlations of plasma BDNF with HDRS and BPRS scores were found for all depressive patients [59].

While plasma BDNF levels in MDD patients with a suicide attempt were significantly lower than those in nonsuicidal MDD patients (supplementary data), results also highlighted the correlation of lower BDNF among depressed patients with recurring depressive episodes, and between lower BDNF and the non-psychotic subtype of depression [59].
In terms of serum and plasma BDNF decline in major depression and its relation with the reduction of BDNF stored in platelets, Lee and Kim examined the change of platelet BDNF levels in MDD and normal controls and their correlation with recent suicide attempt/s and depressed episode recurrence [60]. The study measurements included the HDRS, the Montgomery-Asberg Depression Rating Scale (MADRS), platelet - rich plasma (PRP), and platelet-poor plasma (PPP) and mean platelet BDNF content (mPPT) [60]. In this study, platelet BDNF contents were decreased in patients with MDD compared to the control group, and lower PRP BDNF levels were found in non-suicidal and suicidal patients compared to healthy controls $(\mathrm{F}=(2.57)=3.775, \mathrm{P}$ $=0.029$ ) [60].

The clinical feature of a recent suicide attempt did not differentiate between the MDD sample and the control group, in regards to mPPT BDNF $(\mathrm{t}=-0.109, \mathrm{P}=0.914)$, HDRS (non suicidal MDD: $23.7 \pm 5.3$ vs. suicidal MDD $20.9 \pm 6.0, \mathrm{t}=1.171, \mathrm{df}=58, \mathrm{P}=0.254)$ and MADRS scores (non suicidal MDD: $30.3 \pm 8.4$ vs. suicidal MDD $30.6 \pm 8.7$, $\mathrm{t}=-0.084, \mathrm{df}=58, \mathrm{P}=0.934)[60]$. The results indicated that $\mathrm{mPPT}$ BDNF was significantly lower in those with recurrent-episode depression than those with a first episode (P $=0.033$ ), and no significant difference between non-suicidal and suicidal MDD patients was found [60]. The authors suggest that mPPT BDNF may reflect a tendency towards MDD recurrence rather than suicide risk in MDD patients [60].

\section{Discussion}

This review has examined existing studies on blood BDNF levels and suicidal behaviour among patients with psychiatric disorders. While major depressive disorder was the main psychiatric disorder investigated in relation to BDNF regulation, four out of the seven reviewed papers focused on depressed patients were conducted from the same Korean research group [57-60]. Patients with a history of suicide attempts have decreased BDNF levels in all studies with depression and adjustment disorders, but not schizophrenia. Furthermore the lethality of suicide attempts (measured with the Weisman and Worden's RRR system and LSARSII scale) did not show any significant difference in terms of the deregulated blood BDNF levels among suicidal patients. Genetic studies have noted that BDNF Val66Met polymorphism has an effect on the lethality of suicide attempts based on both genders, while post-mortem findings have suggested a sex effect on the BDNF levels of suicide victim [10, 12, 40]. However the studies reviewed here did not find any effect of either gender or lethality of attempts on blood BDNF levels within the suicidal study groups. As a limitation, it should be noted that, overall, the studies that grouped the patients according to suicide history did not present or measure the number of previous suicide attempts of their study sam- 
ples, a variable which could have an effect on blood BDNF deregulation.

In terms of the psychiatric disorders tested for blood BDNF differences, the main focus was on MDD, and implications for the potential regulating role of depression and related suicide history on BDNF levels, were sought. With regard to the identification of biological markers of depression, a probable association of the decreased sBDNF levels with the reduced platelet BDNF contents among MDD patients has been previously implied, while sBDNF has been suggested as a biomarker for antidepressant efficacy [5, 60, 61]. Furthermore, a trend in the levels of the depression severity in the reviewed studies, measured through the HDRS and in relation to the levels of BDNF, has not been seen.

While no association was found between BDNF protein levels and suicide history in Huang et al. study, research outcomes regarding schizophrenia are mixed in regards to the sBDNF levels when compared to healthy controls [14, $52,53]$. While suicidality has not been explored sufficiently among patients with schizophrenia in blood studies, and while there is no consistent evidence of the sBDNF levels in schizophrenia, sBDNF has been indicated as a non significant biological marker of suicidal behaviour in schizophrenic patients [51, 52].

\section{Implications}

A proximate relationship between BDNF protein levels in blood and suicide history, among patients with psychiatric disorders, has been evident in the majority of studies, and the neurotrophin role in suicide risk has been implied. Consistency has not been evident between blood and a variety of genetic studies with regard to BDNF correlation with lethality of attempts and gender [10,12, 40], leaving aside that no blood study has used gender comparison as a measurement outcome in BDNF protein levels. Considering the determinants of blood BDNF in different populations, which blood sample type (plasma, serum, platelet) is reliable when examining BDNF protein levels among people with mental illness, has yet to be determined [62].

As neurotrophins are stress-regulated, and since prolonged stress related with recent suicide attempts could be potentially associated with decreased BDNF levels, future blood BDNF studies should take into account the number of attempts with each specified suicide method [54]. Including inpatient samples with more than one suicide attempt and method may show differences in decreased blood BDNF levels and their correlation with the lethality of suicide attempt and gender. Furthermore, potential gender differences could be revealed in blood studies, as both sera and platelets have been proposed in regards to the BDNF content based on sex $[55,58,62]$.

Given the genetic findings of the decreased BDNF expression in suicidal patients $[22,30,31]$ and the clinical re- flection of cortical and hippocampal BDNF levels in blood, the decreased blood BDNF levels in patients at suicide risk apparently derived from this review could be of great significance. No study to date has measured and presented any correlation between the BDNF polymorphism Val66Met and the blood BDNF protein levels, in terms of suicidal history, within the same study group of psychiatric patients. Future studies focusing on BDNF polymorphisms among patients with previous suicide attempts, should include such a strategy since the BDNF polymorphism Val66Met has been indicated as a polymorphism highly contributing to suicidal behaviour [31]. Presenting potential positive correlations between BDNF protein levels and the latter would add in the evidence of BDNF's regulating role in suicidal behaviour and the reflection of brain BDNF in blood.

Differentiating psychiatric patients at suicide risk based on their decreased levels of blood BDNF could be a potential tool in suicide prevention, taking into account that specific pharmacological compounds, capable of increasing BDNF levels of patients with mental illness, could be used [1, 24, 31]. Consequently, further studies are required in regards to a potential blood BDNF threshold among patients at suicide risk. A blood BDNF cut-off point of suicide risk among patients with mood disorders has not been investigated, with the exception of one study in this review [58]. The role of biological markers measured in blood could be of importance in clinical practice and suicide prevention, as no biological distinction to date provides a clear picture of patients most at risk of suicide.

Despite increasing attention on the pathophysiology of suicidal behaviour, no clear evidence has been found on BDNF's role as a biological marker of suicidality, although a direct link between BDNF down regulation and suicide pathogenesis is being actively pursued $[25,31,39,56,58$, $63,64]$. Further studies should examine blood BDNF levels among psychiatric patients who use different suicide attempt methods and with differences in the number of previous suicide attempts, while more focus should be given to the potential blood BDNF threshold among patients at suicide risk. Replication studies are needed in regard to the reflection of brain BDNF in blood and the role of BDNF in major stressrelated disorders and suicidality.

\section{Conflict of Interest}

The authors declare that they have no conflict of interest.

\section{Abbreviations}

BDNF, brain-derived neurotrophic factor; sBDNF, serum brain-derived neurotrophic factor; MDD, major depressive disorders. 


\section{References}

1. Duman RS, Monteggia LM. A neurotrophic model for stress-related mood disorders. Biol Psychiatry. 2006;59(12):1116-1127.

2. Gold PW, Chrousos GP. Organization of the stress system and its dysregulation in melancholic and atypical depression: high vs low CRH/NE states. Mol Psychiatry. 2002;7(3):254-275.

3. Neves FS, Malloy-Diniz L, Romano-Silva MA, Campos SB, Miranda DM, De Marco L, Figueira PG, et al. The role of BDNF genetic polymorphisms in bipolar disorder with psychiatric comorbidities. J Affect Disord. 2011;131(1-3):307-311.

4. Perroud N, Courtet P, Vincze I, Jaussent I, Jollant F, Bellivier F, Leboyer $\mathrm{M}$, et al. Interaction between BDNF Val66Met and childhood trauma on adult's violent suicide attempt. Genes Brain Behav. 2008;7(3):314-322.

5. Sen S, Duman R, Sanacora G. Serum brain-derived neurotrophic factor, depression, and antidepressant medications: meta-analyses and implications. Biol Psychiatry. 2008;64(6):527-532.

6. Wagner S, Baskaya O, Dahmen N, Lieb K, Tadic A. Modulatory role of the brain-derived neurotrophic factor Val66Met polymorphism on the effects of serious life events on impulsive aggression in borderline personality disorder. Genes Brain Behav. 2010;9(1):97-102.

7. Hwang JP, Tsai SJ, Hong CJ, Yang CH, Lirng JF, Yang YM. The Val66Met polymorphism of the brain-derived neurotrophic-factor gene is associated with geriatric depression. Neurobiol Aging. 2006;27(12):1834-1837.

8. Kauer-Sant'Anna M, Tramontina J, Andreazza AC, Cereser K, da Costa S, Santin A, Yatham LN, et al. Traumatic life events in bipolar disorder: impact on BDNF levels and psychopathology. Bipolar Disord. 2007;9(Suppl 1):128-135.

9. Kohli MA, Salyakina D, Pfennig A, Lucae S, Horstmann S, Menke A, Kloiber S, et al. Association of genetic variants in the neurotrophic receptor-encoding gene NTRK2 and a lifetime history of suicide attempts in depressed patients. Arch Gen Psychiatry. 2010;67(4):348-359.

10. Kozicz T, Tilburg-Ouwens D, Faludi G, Palkovits M, Roubos E. Gender-related urocortin 1 and brain-derived neurotrophic factor expression in the adult human midbrain of suicide victims with major depression. Neuroscience. 2008;152(4):1015-1023.

11. Lee BH, Kim YK. BDNF mRNA expression of peripheral blood mononuclear cells was decreased in depressive patients who had or had not recently attempted suicide. J Affect Disord. 2010;125(1-3):369-373.

12. Schenkel LC, Segal J, Becker JA, Manfro GG, Bianchin MM, Leistner-Segal S. The BDNF Val66Met polymorphism is an independent risk factor for high lethality in suicide attempts of depressed patients. Prog Neuropsy- chopharmacol Biol Psychiatry. 2010;34(6):940-944.

13. de Luca V, Souza RP, Zai CC, Panariello F, Javaid N, Strauss J, Kennedy JL, et al. Parent of origin effect and differential allelic expression of BDNF Val66Met in suicidal behaviour. World J Biol Psychiatry. 2011;12(1):4247.

14. Xiu MH, Hui L, Dang YF, Hou TD, Zhang CX, Zheng YL, Chen da C, et al. Decreased serum BDNF levels in chronic institutionalized schizophrenia on longterm treatment with typical and atypical antipsychotics. Prog Neuropsychopharmacol Biol Psychiatry. 2009;33(8):1508-1512.

15. Castren E. Is mood chemistry? Nat Rev Neurosci. 2005;6(3):241-246.

16. Chen L, Lawlor DA, Lewis SJ, Yuan W, Abdollahi MR, Timpson NJ, Day IN, et al. Genetic association study of BDNF in depression: finding from two cohort studies and a meta-analysis. Am J Med Genet B Neuropsychiatr Genet. 2008;147B(6):814-821.

17. Grunblatt E, Hupp E, Bambula M, Zehetmayer S, Jungwirth S, Tragl KH, Fischer P, et al. Association study of BDNF and CNTF polymorphism to depression in nondemented subjects of the "VITA" study. J Affect Disord. 2006;96(1-2):111-116.

18. Karege F, Perret G, Bondolfi G, Schwald M, Bertschy G, Aubry JM. Decreased serum brain-derived neurotrophic factor levels in major depressed patients. Psychiatry Res. 2002;109(2):143-148.

19. Tsai SJ, Cheng CY, Yu YW, Chen TJ, Hong CJ. Association study of a brain-derived neurotrophic-factor genetic polymorphism and major depressive disorders, symptomatology, and antidepressant response. Am J Med Genet B Neuropsychiatr Genet. 2003;123B(1):19-22.

20. Nibuya M, Morinobu S, Duman RS. Regulation of BDNF and trkB mRNA in rat brain by chronic electroconvulsive seizure and antidepressant drug treatments. J Neurosci. 1995;15(11):7539-7547.

21. Karege F, Vaudan G, Schwald M, Perroud N, La Harpe R. Neurotrophin levels in postmortem brains of suicide victims and the effects of antemortem diagnosis and psychotropic drugs. Brain Res Mol Brain Res. 2005;136(12):29-37.

22. Dwivedi Y. Brain-derived neurotrophic factor and suicide pathogenesis. Ann Med. 2010;42(2):87-96.

23. Kobayashi K, Shimizu E, Hashimoto K, Mitsumori M, Koike K, Okamura N, Koizumi H, et al. Serum brain-derived neurotrophic factor (BDNF) levels in patients with panic disorder: as a biological predictor of response to group cognitive behavioral therapy. Prog Neuropsychopharmacol Biol Psychiatry. 2005;29(5):658-663.

24. Schmidt HD, Duman RS. Peripheral BDNF produces antidepressant-like effects in cellular and behavioral models. Neuropsychopharmacology. 2010;35(12):23782391. 
25. Shimizu E, Hashimoto K, Okamura N, Koike K, Komatsu N, Kumakiri C, Nakazato M, et al. Alterations of serum levels of brain-derived neurotrophic factor (BDNF) in depressed patients with or without antidepressants. Biol Psychiatry. 2003;54(1):70-75.

26. Dwivedi Y. Brain-derived neurotrophic factor: role in depression and suicide. Neuropsychiatr Dis Treat. 2009;5:433-449.

27. Kim B, Kim CY, Hong JP, Kim SY, Lee C, Joo YH. Brain-derived neurotrophic factor Val/Met polymorphism and bipolar disorder. Association of the Met allele with suicidal behavior of bipolar patients. Neuropsychobiology. 2008;58(2):97-103.

28. Pandey GN, Dwivedi Y. What can post-mortem studies tell us about the pathoetiology of suicide? Future Neurol. 2010;5(5):701-720.

29. Sarchiapone M, Carli V, Roy A, Iacoviello L, Cuomo C, Latella MC, di Giannantonio M, et al. Association of polymorphism (Val66Met) of brain-derived neurotrophic factor with suicide attempts in depressed patients. Neuropsychobiology. 2008;57(3):139-145.

30. Sher L. Brain-derived neurotrophic factor and suicidal behavior. QJM. 2011;104(5):455-458.

31. Zai CC, Manchia M, De Luca V, Tiwari AK, Chowdhury NI, Zai GC, Tong RP, et al. The brain-derived neurotrophic factor gene in suicidal behaviour: a meta-analysis. Int J Neuropsychopharmacol. 2012;15(8):10371042.

32. Iga J, Ueno S, Yamauchi K, Numata S, Tayoshi-Shibuya S, Kinouchi S, Nakataki M, et al. The Val66Met polymorphism of the brain-derived neurotrophic factor gene is associated with psychotic feature and suicidal behavior in Japanese major depressive patients. Am J Med Genet B Neuropsychiatr Genet. 2007;144B(8):10031006.

33. Zarrilli F, Angiolillo A, Castaldo G, Chiariotti L, Keller S, Sacchetti S, Marusic A, et al. Brain derived neurotrophic factor (BDNF) genetic polymorphism (Val$66 \mathrm{Met}$ ) in suicide: a study of 512 cases. Am J Med Genet B Neuropsychiatr Genet. 2009;150B(4):599-600.

34. Dwivedi Y, Rizavi HS, Conley RR, Roberts RC, Tamminga CA, Pandey GN. Altered gene expression of brain-derived neurotrophic factor and receptor tyrosine kinase B in postmortem brain of suicide subjects. Arch Gen Psychiatry. 2003;60(8):804-815.

35. Pandey GN. Neurobiology of Adult and Teenage Suicide. Asian J Psychiatr. 2011;4(1):2-13.

36. Pandey GN, Ren X, Rizavi HS, Conley RR, Roberts RC, Dwivedi Y. Brain-derived neurotrophic factor and tyrosine kinase B receptor signalling in post-mortem brain of teenage suicide victims. Int J Neuropsychopharmacol. 2008;11(8):1047-1061.

37. Ozan E, Okur H, Eker C, Eker OD, Gonul AS, Akarsu $\mathrm{N}$. The effect of depression, BDNF gene val66met poly- morphism and gender on serum BDNF levels. Brain Res Bull. 2010;81(1):61-65.

38. Schrijvers DL, Bollen J, Sabbe BG. The gender paradox in suicidal behavior and its impact on the suicidal process. J Affect Disord. 2012;138(1-2):19-26.

39. Verhagen M, van der Meij A, van Deurzen PA, Janzing JG, Arias-Vasquez A, Buitelaar JK, Franke B. Metaanalysis of the BDNF Val66Met polymorphism in major depressive disorder: effects of gender and ethnicity. Mol Psychiatry. 2010;15(3):260-271.

40. Pregelj P, Nedic G, Paska AV, Zupanc T, Nikolac M, Balazic J, Tomori M, et al. The association between brain-derived neurotrophic factor polymorphism (BDNF Val66Met) and suicide. J Affect Disord. 2011;128(3):287-290.

41. Shalev I, Lerer E, Israel S, Uzefovsky F, Gritsenko I, Mankuta D, Ebstein RP, et al. BDNF Val66Met polymorphism is associated with HPA axis reactivity to psychological stress characterized by genotype and gender interactions. Psychoneuroendocrinology. 2009;34(3):382-388.

42. Sohrabji F, Lewis DK. Estrogen-BDNF interactions: implications for neurodegenerative diseases. Front Neuroendocrinol. 2006;27(4):404-414.

43. Young EA, Becker JB. Perspective: sex matters: gonadal steroids and the brain. Neuropsychopharmacology. 2009;34(3):537-538.

44. Luo KR, Hong CJ, Liou YJ, Hou SJ, Huang YH, Tsai SJ. Differential regulation of neurotrophin S100B and BDNF in two rat models of depression. Prog Neuropsychopharmacol Biol Psychiatry. 2010;34(8):1433-1439.

45. Pan W, Banks WA, Fasold MB, Bluth J, Kastin AJ. Transport of brain-derived neurotrophic factor across the blood-brain barrier. Neuropharmacology. 1998;37(12):1553-1561.

46. Karege F, Schwald M, Cisse M. Postnatal developmental profile of brain-derived neurotrophic factor in rat brain and platelets. Neurosci Lett. 2002;328(3):261-264.

47. Gass P, Hellweg R. Peripheral brain-derived neurotrophic factor (BDNF) as a biomarker for affective disorders? Int J Neuropsychopharmacol. 2010;13(1):1-4.

48. Pillai A, Kale A, Joshi S, Naphade N, Raju MS, Nasrallah H, Mahadik SP. Decreased BDNF levels in CSF of drug-naive first-episode psychotic subjects: correlation with plasma BDNF and psychopathology. Int J Neuropsychopharmacol. 2010;13(4):535-539.

49. Elfving B, Plougmann PH, Muller HK, Mathe AA, Rosenberg R, Wegener G. Inverse correlation of brain and blood BDNF levels in a genetic rat model of depression. Int J Neuropsychopharmacol. 2010;13(5):563572.

50. Klein AB, Williamson R, Santini MA, Clemmensen C, Ettrup A, Rios M, Knudsen GM, et al. Blood BDNF concentrations reflect brain-tissue BDNF levels across 
species. Int J Neuropsychopharmacol. 2011;14(3):347353.

51. Shimizu E, Hashimoto K, Watanabe H, Komatsu N, Okamura N, Koike K, Shinoda N, et al. Serum brain-derived neurotrophic factor (BDNF) levels in schizophrenia are indistinguishable from controls. Neurosci Lett. 2003;351(2):111-114.

52. Toyooka K, Asama K, Watanabe Y, Muratake T, Takahashi M, Someya T, Nawa H. Decreased levels of brainderived neurotrophic factor in serum of chronic schizophrenic patients. Psychiatry Res. 2002;110(3):249-257.

53. Huang TL, Lee CT. Associations between serum brainderived neurotrophic factor levels and clinical phenotypes in schizophrenia patients. J Psychiatr Res. 2006;40(7):664-668.

54. Deveci A, Aydemir O, Taskin O, Taneli F, Esen-Danaci A. Serum BDNF levels in suicide attempters related to psychosocial stressors: a comparative study with depression. Neuropsychobiology. 2007;56(2-3):93-97.

55. Lommatzsch M, Zingler D, Schuhbaeck K, Schloetcke K, Zingler C, Schuff-Werner P, Virchow JC. The impact of age, weight and gender on BDNF levels in human platelets and plasma. Neurobiol Aging. 2005;26(1):115123.

56. Dawood T, Anderson J, Barton D, Lambert E, Esler M, Hotchkin E, Haikerwal D, et al. Reduced overflow of BDNF from the brain is linked with suicide risk in depressive illness. Mol Psychiatry. 2007;12(11):981-983.

57. Kim YK., Won SD, Hur JW, Lee BH, Lee HY, Shim SH, Han, SW et al. Exploration of biological markers of sui- cidal behavior in major depressive disorder. Psychiatry Investigation. 2007(a);4(1):13-21.

58. Kim YK, Lee HP, Won SD, Park EY, Lee HY, Lee BH, Lee SW, et al. Low plasma BDNF is associated with suicidal behavior in major depression. Prog Neuropsychopharmacol Biol Psychiatry. 2007;31(1):78-85.

59. Lee BH, Kim H, Park SH, Kim YK. Decreased plasma BDNF level in depressive patients. J Affect Disord. 2007;101(1-3):239-244.

60. Lee BH, Kim YK. Reduced platelet BDNF level in patients with major depression. Prog Neuropsychopharmacol Biol Psychiatry. 2009;33(5):849-853.

61. Brunoni AR, Lopes M, Fregni F. A systematic review and meta-analysis of clinical studies on major depression and BDNF levels: implications for the role of neuroplasticity in depression. Int J Neuropsychopharmacol. 2008;11(8):1169-1180.

62. Bus BA, Molendijk ML, Penninx BJ, Buitelaar JK, Kenis G, Prickaerts J, Elzinga BM, et al. Determinants of serum brain-derived neurotrophic factor. Psychoneuroendocrinology. 2011;36(2):228-239.

63. Tan YL, Zhou DF, Cao LY, Zou YZ, Zhang XY. Decreased BDNF in serum of patients with chronic schizophrenia on long-term treatment with antipsychotics. Neurosci Lett. 2005;382(1-2):27-32.

64. Aydemir C, Yalcin ES, Aksaray S, Kisa C, Yildirim SG, Uzbay T, Goka E. Brain-derived neurotrophic factor (BDNF) changes in the serum of depressed women. Prog Neuropsychopharmacol Biol Psychiatry. 2006;30(7):1256-1260. 